\title{
A time for trust and transparency
}

\author{
Nature Astronomy is committed to open science. The new open access option for authors is the next step along the \\ road to full transparency, reproducibility and accessibility.
}

$\mathrm{B}$ ack in the December Editorial, we announced that Nature Astronomy, along with Nature and all the

Nature Research journals, would become transformative journals from 1 January 2021. Practically, this now means that authors wishing to make their papers free to access at the point of publication and to give anyone the right to share, use and build on the work for free (under a Creative Commons licence) can do so by paying an article processing charge (APC). The APC (set at $€ 9,500$ ) may be covered by the funding body, if they stipulate open access publication; alternatively it may be covered by an author's institution under a 'transformative agreement', such as the Nature journals' read-and-publish deal with the Max Planck Digital Library.

Under the transformative journal model, the traditional subscription route to publishing remains an option: authors pay no fees, and readers are charged for access to the content through institutional site licences, for instance. Authors who do not, as yet, have access to funding for APCs are able to publish in Nature Astronomy exactly as before. But being transformative means that Nature Astronomy is fully committed to increasing the proportion of open access papers it publishes over time, with the aim of becoming a fully open access journal in the future; we will have transparent growth targets for open access content that are in line with the principles of cOAlition S.

The potential benefits of this change are clear: open access papers are downloaded and cited more frequently. The effect of making research free for all was best exemplified during the current pandemic, when all relevant COVID-19 research published by Nature Research was made available for free to help scientists, policymakers and (one would hope) lawmakers make use of the latest research in combatting the virus and its deadly wake.

Although proponents of open access publishing welcome this change to our business model, it is noted that our APC is higher than that charged by other publishers. But it reflects the costs associated with producing such highly selective journals, staffed with professional editors rather than academic editors. Across the portfolio, the acceptance rate at Nature and the Nature Research journals is $8 \%$; editors spend roughly $60 \%$ of their time on papers that they ultimately do not end up publishing. And once papers are accepted, we have dedicated in-house copyeditors and art editors to improve the clarity and quality of presentation; other colleagues work to engage with the research community and the wider media. This infographic provides a high-level view of what goes on inside Nature.

Of course, one way to reduce the cost per author for open access publication would be to publish more papers. However, readers appreciate having a carefully curated collection of research, as it is becoming increasingly difficult to keep up with and parse through growing numbers of publications. Moreover, in the ongoing push for governments tackling pandemics, climate change and other large-scale challenges to follow evidence-based research, it is important to have a focused set of high-quality discoveries, analyses and recommendations that they know they can trust. Without personal research goals or academic agendas, our full-time editors are unbiased and can ensure a robust peer-review process involving as diverse a group of experts as possible across the globe.

So what does the change to our publishing model mean for our authors? First of all, there is more choice for them, whatever their funding situation. And the editorial process remains the same. As always, editors are impartial to any financial incentives and, to maintain that impartiality, the decision to publish a paper as open access will be made by the corresponding author only after acceptance of a paper. We offer assistance to authors to both discover and apply for funding, but ultimately, the onus should be on the authors to check. If any of the co-authors is supported by a funding body requiring open access, then the paper must be published open access. In such cases, the funding should come from a central source rather than individual research grants.

Should authors choose to make their papers open access, even if their funders do not require it, they may do so at acceptance as well. The motivation will vary from community to community and open access is but one facet of open science. Having open data is particularly important when it comes to collaboration and collective learning. Take, for example, the James Webb Space Telescope (JWST) and its Director's Discretionary-Early Release Science programme. Those chosen proposals will take place during the first five months following commissioning, and there will be no exclusive access period: "both raw and pipeline-processed data will enter the public domain immediately after processing and validation at [the Space Telescope Science Institute]". This policy will allow the entire community to quickly learn about JWST's instruments and capabilities, so that it will be able to make full use of the observatory in the upcoming proposal cycles. And more to the point, any scientific breakthroughs would be discovered almost immediately.

In the spirit of maximum transparency, reproducibility and accessibility - allowing for greater dissemination and collaboration - Nature Research has introduced technical checklists, extended data and tables, raw data, data and code accessibility statements, and, in some cases, the referee reports are published alongside the papers. Becoming transformative is the next step. The road to fully open but also reliable, reproducible and inclusive science is not a smooth one and it will take time, but we are taking steps in the right direction and we are ready to work together with the community towards this important end goal.

Published online: 12 February 2021 https://doi.org/10.1038/s41550-021-01319-x 